Research Paper

\title{
GSK1059615 kills head and neck squamous cell carcinoma cells possibly via activating mitochondrial programmed necrosis pathway
}

\author{
Jing Xie ${ }^{1}$, Quan $\mathbf{L i}^{2}$, Xi Ding ${ }^{1}$ and Yunyun Gao $^{1}$ \\ ${ }^{1}$ Department of Stomatology, The First Affiliated Hospital of Wenzhou Medical University, Wenzhou, China \\ ${ }^{2}$ Center of Stomatology, The Second Affiliated Hospital of Soochow University, Suzhou, China \\ Correspondence to: Jing Xie, email: xiejingwenzhou9@163.com \\ Keywords: HNSCC, PI3K-AKT-mTOR, GSK1059615, programmed necrosis \\ Received: November 23, $2016 \quad$ Accepted: January 11, $2017 \quad$ Published: February 07, 2017 \\ Copyright: Xie et al. This is an open-access article distributed under the terms of the Creative Commons Attribution License 3.0 (CC \\ BY 3.0), which permits unrestricted use, distribution, and reproduction in any medium, provided the original author and source are \\ credited.
}

\section{ABSTRACT}

This study tested the anti-head and neck squamous cell carcinoma (HNSCC) cell activity by GSK1059615, a novel PI3K and mTOR dual inhibitor. GSK1059615 inhibited survival and proliferation of established (SCC-9, SQ20B and A253 lines) and primary human HNSCC cells. GSK1059615 blocked PI3K-AKT-mTOR activation in HNSCC cells. Intriguingly, GSK1059615 treatment in HNSCC cells failed to provoke apoptosis, but induced programmed necrosis. The latter was tested by mitochondria depolarization, ANT-1-cyclophilin-D mitochondrial association and lactate dehydrogenase (LDH) release. Reversely, mPTP blockers (sanglifehrin A, cyclosporin A and bongkrekic acid) or cyclophilin-D shRNA dramatically alleviated GSK1059615-induced SCC-9 cell death. Further studies demonstrated that GSK1059615 i.p. injection suppressed SCC9 tumor growth in nude mice, which was compromised with co-administration with cyclosporin A. Thus, targeting PI3K-AKT-mTOR pathway by GSK1059615 possibly provokes programmed necrosis pathway to kill HNSCC cells.

\section{INTRODUCTION}

Head and neck squamous cell carcinoma (HNSCC) is a large heterogeneous family of carcinomas, including carcinomas of face, nasopharynx, oral cavity, and larynx [1-3]. The overall survival is still poor for those with high-degree or malignant tumors [1-4]. One major signaling that is often dysregulated in HNSCC is phosphatidylinositol 3-kinase-AKT-mammalian target of rapamycin (PI3K-AKT-mTOR) cascade [5-7]. Recent research efforts have developed a novel PI3K and mTOR dual inhibitor, named GSK1059615 [8]. As compared to other PI3K-AKT-mTOR specific inhibitors, the advantage of this compound is significant, as it simantanuously blocks PI3K and mTOR [8]. Therefore, it has the potential to completely shut down the PI3K-AKT-mTOR cascade [8]. The potential effect of GSK1059615 on HNSCC cells is evaluated in this preclinical study.

Recent studies have proposed a non-apoptotic form of cell death named programmed necrosis, which also requires mitochondrial permeability transition pore
(mPTP) opening [9-13]. The channel complex mPTP has several major components, including voltage-dependent anion channel (VDAC, in outer membrane), the adenine nucleotide translocator-1 (ANT-1, in the inner membrane), cyclophilin-D (in the matrix) [14] [14-16]. Many cancerkilling agents are capable of inducing mitochondrial depolarization and ANT-1-cyclophilin-D association, causing mPTP opening and subsequent cell necrosis (but not apoptosis) [9-13]. In the present study, we show that GSK1059615 also provokes programmed necrosis pathway in HNSCC cells.

\section{RESULTS}

GSK1059615 is cytotoxic to both established and primary human HNSCC cells

First, we tested the potential effect of GSK1059615 on HNSCC cell survival. SCC-9 cells, cultured in complete medium (with $10 \% \mathrm{FBS}$ ), were treated with gradually increasing concentration $(0.3-30 \mu \mathrm{M})$ of GSK1059615, 
and tetrazolium dye (MTT) assay was applied to test cell survival. Results in Figure 1A demonstrated that treatment with GSK1059615 in SCC-9 cells dosedependently inhibited cell survival. The MTT OD was significantly decreased following GSK1059615 (1-30 $\mu \mathrm{M}$ ) treatment (Figure 1A). Notably, it took at least $48 \mathrm{~h}$ for the GSK1059615 treatment to exert significant antisurvival activity (Figure 1A). The IC50 of GSK1059615, or the concentration that inhibited $50 \%$ of cell survival, was close to $3 \mu \mathrm{M}$ ( $72 \mathrm{~h}$ and $96 \mathrm{~h}$ treatment duration) (Figure 1A). To further confirm the anti-survival activity by GSK1059615, clonogenicity assay was performed. Results in Figure 1B showed that GSK1059615, at 1-30 $\mu \mathrm{M}$, dramatically decreased the number of viable SCC-9 colonies. The lactate dehydrogenase (LDH) content was also significantly increased in the conditional medium of GSK1059615 (1-30 $\mu \mathrm{M})$-treated cells (Figure 1C), implying that GSK1059615 induced SCC-9 cell death.

The activity of GSK1059615 on other HNSCC cells was also tested. In both A253 and SQ20B HNSCC cells [17], GSK1059615 (3 $\mu \mathrm{M}, 72 \mathrm{~h})$ largely decreased cell survival (MTT OD, Figure 1D). On the other hand, the very same GSK1059615 treatment failed to inhibit the survival of two oral epithelial cell lines ("Oepi1/2") (Figure 1D), implying that GSK1059615 could be cytotoxic only to cancer cells. In order to test the effect of GSK1059615 in primary cancer cells, a total of four lines of primary ("patient-derived") oral cavity carcinoma (OCC) cells were established (named "OCC1-4"), which were also treated with GSK1059615 (3 $\mu \mathrm{M}, 72 \mathrm{~h})$. MTT assay results in Figure 1E showed that GSK1059615 was cytotoxic to all the primary cancer cells. Remarkably, we found that GSK1059615 was more potent that other known AKT inhibitors (i.e. LY294002, Wortmannin and perifosine) in killing SCC-9 cells (Figure 1F). Together, these results demonstrate that GSK1059615 is cytotoxic to established and primary human HNSCC cells.

\section{GSK1059615 inhibits human HNSCC cell proliferation}

Cytotoxicity in HNSCC cells could be due to proliferation inhibition. Next, proliferation of GSK1059615-treated HNSCC cells was tested by the BrdU ELISA assay and $\left[\mathrm{H}^{3}\right]$ thymidine incorporation assay [18]. Results from both assays demonstrated clearly

A.

B.

C.
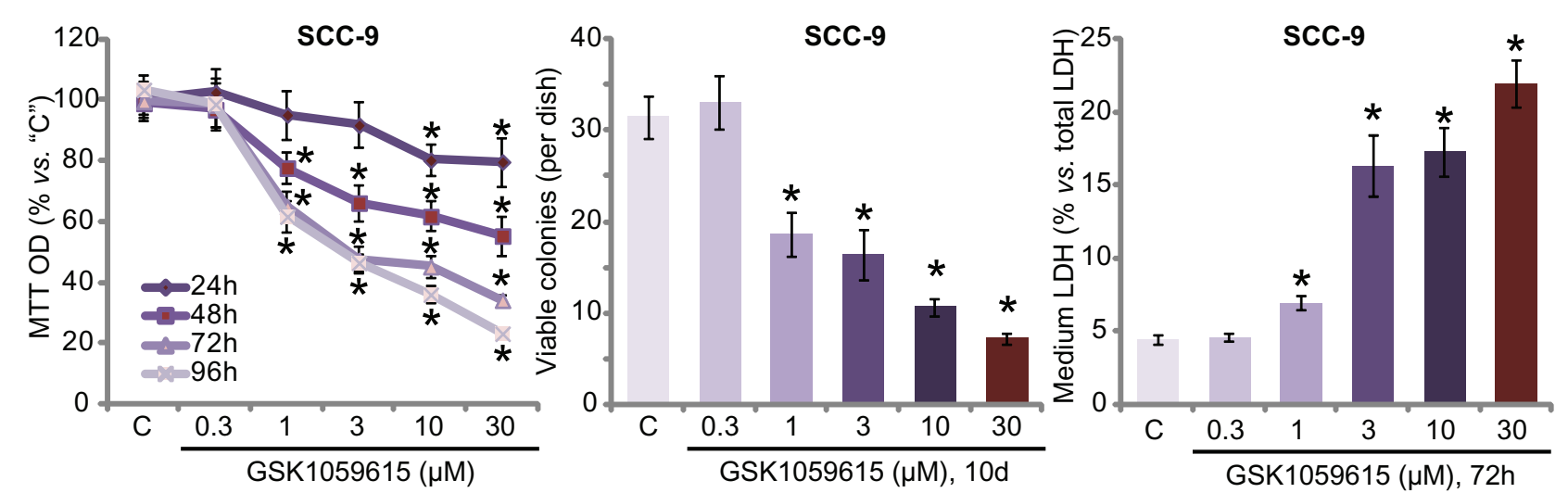

D.
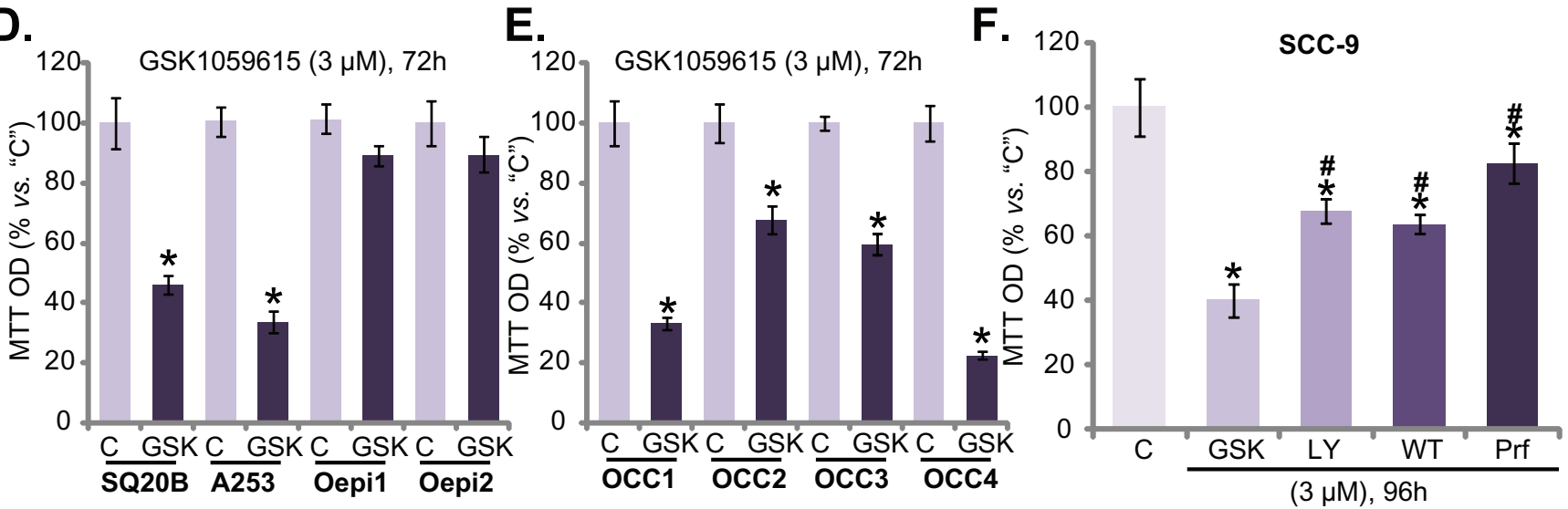

Figure 1: GSK1059615 is cytotoxic to HNSCC cells. HNSCC cell lines (SCC-9, SQ20B and A253) A-D, F., primary human OCC cells ("OCC1-4") E. or oral epithelial cell ("Oepi1/2") (D) were treated with designated concentration of GSK1059615 ("GSK"), or wortmannin ("WT”), LY294002 ("LY") and perifosine ("Prf"), cells were further cultured for indicated time period, and cell survival was tested by listed assays. "C" stands for untreated control group (Same for all Figures). For each assay, $\mathrm{n}=6$. Bars stand for mean \pm SD (Same for all Figures). Experiments in this figure were repeated four times, and similar results were obtained. * $p<0.01$ vs. group "C". 
that GSK1059615 dose-dependently inhibited SCC-9 cell proliferation (Figure 2A and 2B), as the BrdU ELISA OD (Figure $2 \mathrm{~A}$ ) and $\left[\mathrm{H}^{3}\right]$ thymidine incorporation (Figure 2B) were both decreased following GSK1059615 (1-30 $\mu \mathrm{M})$ treatment. Expression of proliferation-associated proteins, including cyclin D1 and cyclin B1, was also significantly downregulated following GSK1059615 (1-10 $\mu \mathrm{M})$ treatment (Figure 2C). Notably, to test cell proliferation, cells were incubated with GSK1059615 for only $24 \mathrm{~h}$, when no significant cytotoxicity was yet noticed (Figure 1A).

BrdU ELISA assay was also performed to test proliferation of other HNSCC cells with GSK1059615 treatment. Results in Figure 2D showed clearly that GSK1059615 $(3 \mu \mathrm{M})$ was anti-proliferative in two other HNSCC cell lines: SQ20B and A253. Yet, the same GSK1059615 treatment failed to inhibit proliferation of oral epithelial cells ("Oepi1/2") (Figure 2D). In the primary OCC cells (all four lines, "OCC1-4"), treatment with GSK1059615 (3 $\mu \mathrm{M}, 24 \mathrm{~h})$ also inhibited cell proliferation, which was again indicated by BrdU ELISA
OD reduction (Figure 2E). Collectively, these results imply that GSK1059615 inhibits human HNSCC cell proliferation.

\section{GSK1059615 blocks PI3K-AKT-mTOR activation in HNSCC cells}

GSK1059615 is a potent PI3K-mTOR duel inhibitor, we thus tested PI3K-AKT-mTOR signaling in GSK1059615-treated cells. The quantified results in Figure $3 \mathrm{~A}$ and $3 \mathrm{~B}$ showed that, treatment with GSK1059615 (3 $\mu \mathrm{M})$ in SCC-9 cells and "OCC1" primary cancer cells dramatically inhibited phosphorylation ("p-") of PI3K p85 (Tyr-458), AKT (Ser-473), mTOR (Ser-2448) and S6K1 (Thr-389). Thus, GSK1059615 apparently blocked PI3K-AKT-mTOR signaling cascade activation in HNSCC cells (Figure 3A and 3B). Remarkably, the basal activation of PI3K-AKT-mTOR cascade was quite low in the oral epithelial cells ("Oepi1") (Figure 3C). p-PI3K p85, p-AKT, p-mTOR and p-S6K1 were almost undetected in the epithelial cells (Figure 3C). These might
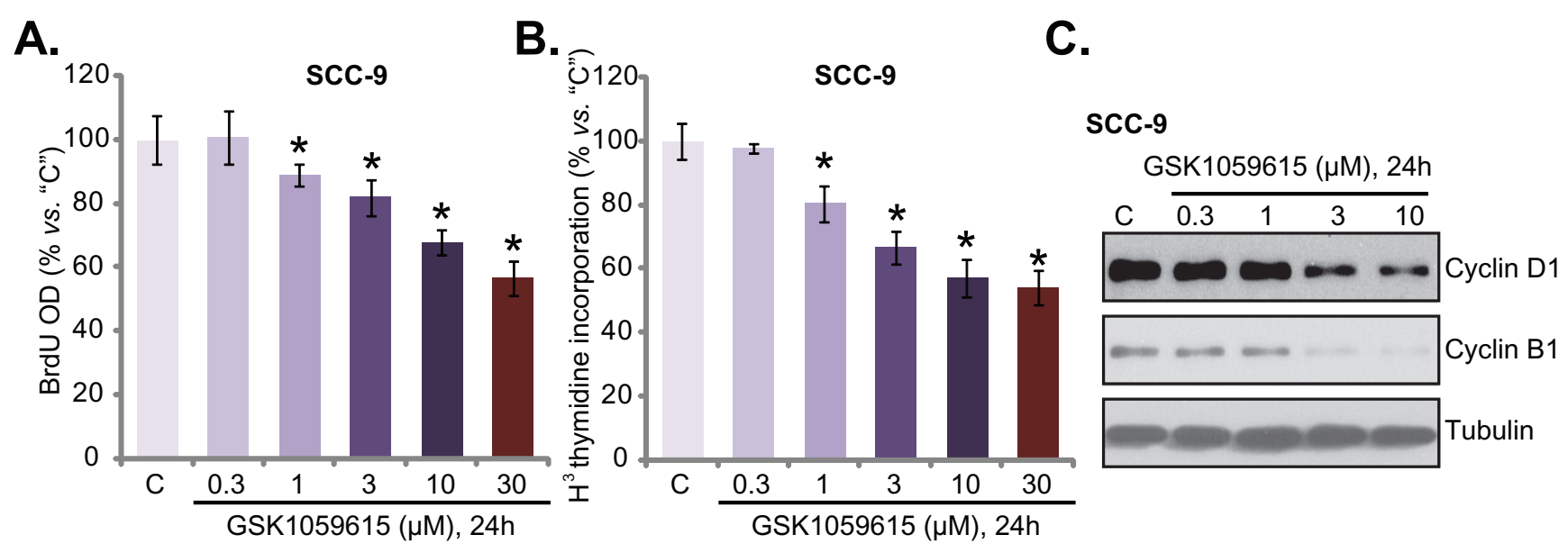

D.

E.
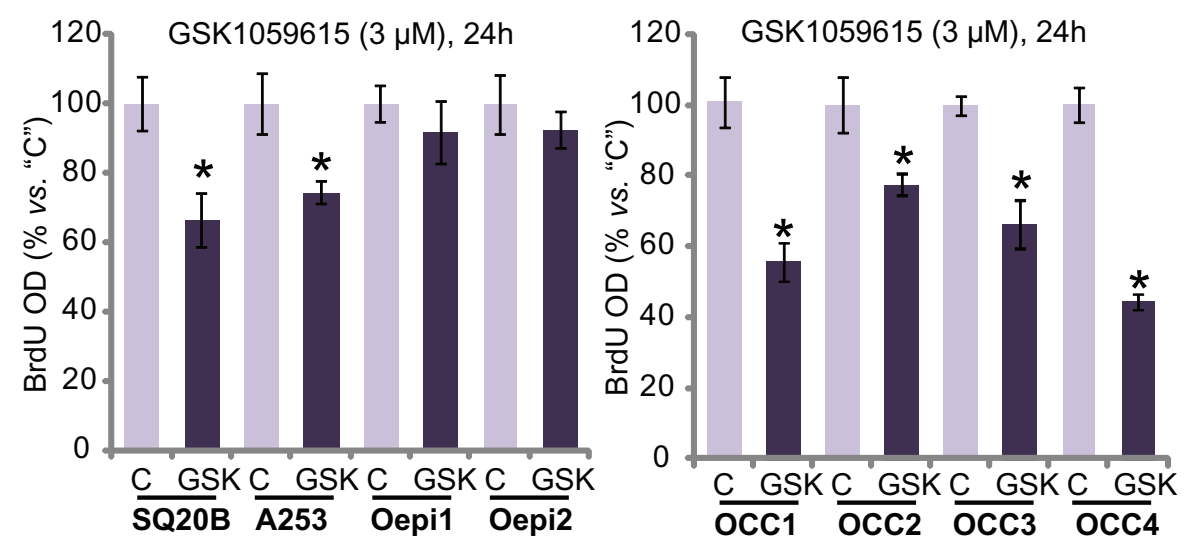

Figure 2: GSK1059615 inhibits HNSCC cell proliferation. HNSCC cell lines (SCC-9, SQ20B and A253) A-D., primary human OCC cells ("OCC1-4") E. or oral epithelial cell ("Oepi1/2") (D) were treated with designated concentration of GSK1059615 ("GSK"), cells were further cultured for indicated time period, cell proliferation was tested by BrdU ELISA assay (A, D and E) and $\left[\mathrm{H}^{3}\right]$ thymidine incorporation assay (B); Expression of proliferation-associated proteins was tested by Western blot assay (C) For each assay, n=5. Experiments in this figure were repeated three times, and similar results were obtained. * $p<0.01$ vs. group "C". 
explain why these epithelial cells were not killed by GSK1059615 (Figure 1). Interestingly, ERK activation, tested by p-ERK1/2 (Thr-202/Tyr-204), was not altered by the same GSK1059615 treatment (Figure 3A-3C). Thus, GSK 1059615 blocks PI3K-AKT-mTOR activation in HNSCC cells.

Next, we compared the activity of GSK1059615 with other PI3K-AKT-mTOR specific inhibitors. Results in Figure 3D and 3E showed that GSK1059615 was significantly more potent in killing SCC-9 cells than same concentration $(3 \mu \mathrm{M})$ of the AKT specific inhibitor MK-2206 [19, 20], mTORC1 inhibitor rapamycin [21] and mTOR kinase inhibitor AZD2014 [17, 22] (Figure 3D and 3E). GSK1059615 resulted in more survival loss (Figure 3D) and cell death (Figure 3E) than the above specific inhibitors.

\section{GSK1059615 fails to provoke apoptosis in HNSCC cells}

We also tested the potential effect of GSK1059615 on cell apoptosis. Three different assays were performed, including the Annexin V FACS assay, Terminal deoxynucleotidyl transferase dUTP nick end labeling (TUNEL) staining assay and Histone DNA apoptosis ELISA assay. To our surprise, the results of these assays showed that GSK1059615 (3 $\mu \mathrm{M}$, the cytotoxic dose) failed to induce significant apoptosis in SCC-9 cells (Figure 4A-4C). Notably, to test cell apoptosis, cells were treated with GSK1059615 for three different time points $(24 \mathrm{~h} / 48 \mathrm{~h} / 72 \mathrm{~h})$, yet no apoptosis activation was noticed at any time point (Figure 4A-4C). On the other hand, gemcitabine, served as a positive control, provoked

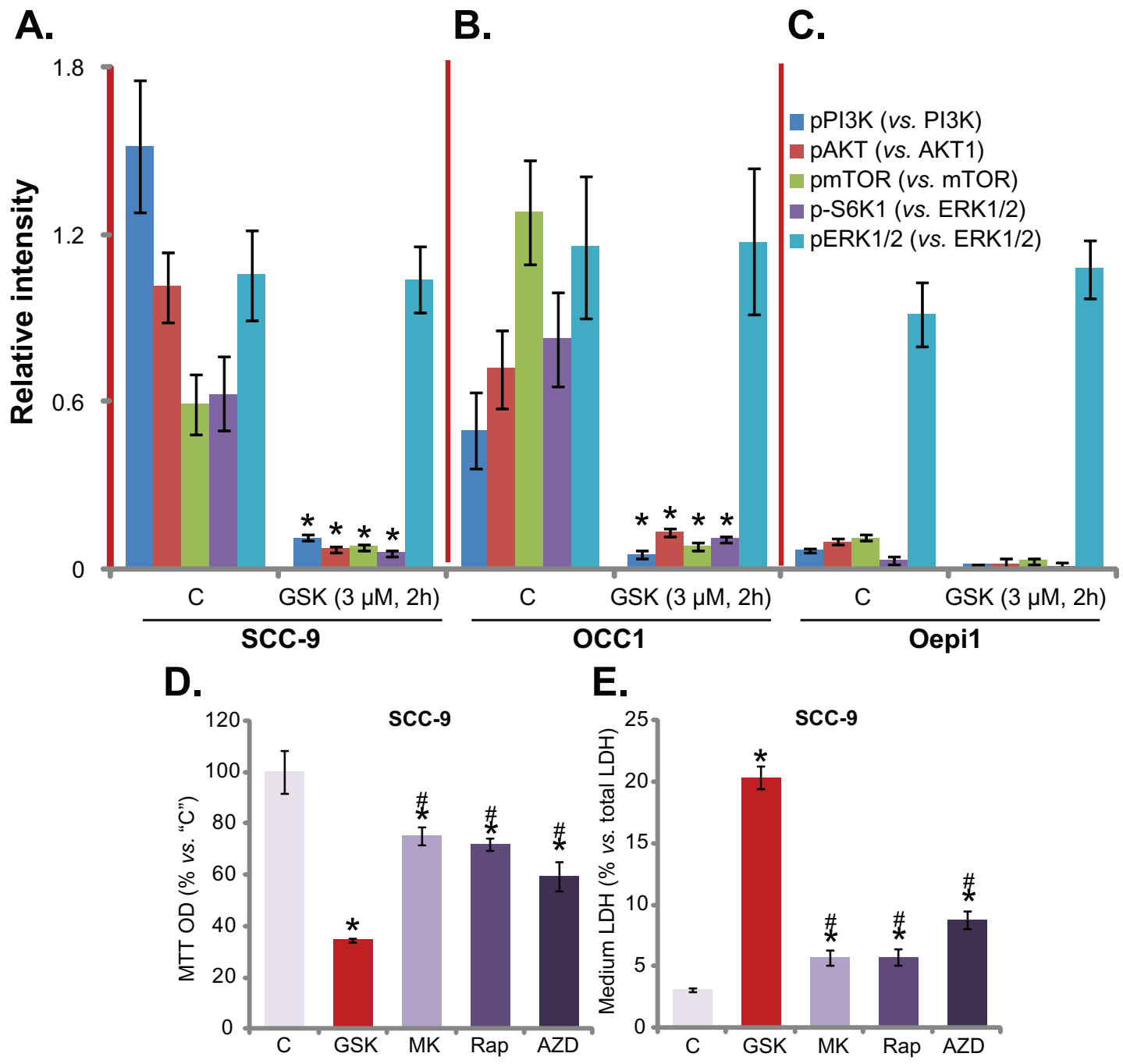

Figure 3: GSK1059615 blocks PI3K-AKT-mTOR activation in HNSCC cells. SCC-9 cells A., primary human OCC cells ("OCC1") B. or oral epithelial cells ("Oepi1") C. were treated with GSK1059615 ("GSK", $3 \mu \mathrm{M}$ ) for 2h, expression of listed kinase proteins in the fresh cell lysates was tested, and data were quantified (three repeats). SCC-9 cells were treated with $3 \mu \mathrm{M}$ of MK-2206 ("MK"), rapamycin ("Rap") or AZD-2014 (“AZD") for 72h, cell viability (MTT assay, D.) and cell death (LDH assay, E.) were tested. Experiments in this figure were repeated three times, and similar results were obtained. * $p<0.01$ vs. group "C". " $p<0.01$ vs. GSK1059615 only (D and E). 
profound apoptosis in SCC-9 cells (Figure 4A-4C). Further studies showed that gemcitabine, but not GSK1059615, induced obvious caspase-3/caspase-9 cleavage in SCC9 cells (Figure 4D). Together, GSK1059615, although is cytotoxic, but fails to induce HNSCC cell apoptosis.

\section{GSK1059615 provokes programmed necrosis in HSNCC cells}

Recent studies have shown that several anti-cancer agents could provoke mitochondrial programmed necrosis pathway to kill cancer cells [9, 12, 23-25]. Our results here also indicated activation of this necrosis pathway by GSK1059615. Mitochondrial co-immunoprecipitation assay ("mito-IP") assay results in Figure 5A showed that GSK1059615 (3 $\mu \mathrm{M})$ in SCC-9 cells induced cyclophilin-D-adenine nucleotide translocator-1 (ANT1) association in the mitochondria, which is the initial step of programmed necrosis pathway activation $[9,12$, 23-25]. Further, GSK1059615 dose-dependently induced mitochondrial membrane potential (MMP) collapse or mPTP opening, which was evidenced by JC-10 fluorescence intensity increase [9, 12, 23-25] (Figure 5B). Remarkably, several mPTP inhibitors, including cyclosporin A [26, 27], sanglifehrin A [26], and bongkrekic acid [28], significantly attenuated GSK1059615-induced SCC-9 cell viability reduction (Figure 5C) and cell death (Figure 5D).

The above results suggest that GSK1059615 provokes programmed necrosis to kill SCC-9 cells. To further this hypothesis, shRNA method was applied to stably knockdown cyclophilin-D, the key component of mPTP. A total of three non-overlapping cyclophilin-D shRNAs (from Dr. Xu [29]) were applied: cyclophilin-D shRNA-1/2/3. All three of them efficiently downregulated cyclophilin-D in SCC-9 cells (Figure 5E). Remarkably, GSK1059615 (3 $\mu \mathrm{M})$-induced cytotoxicity was largely alleviated in the cyclophilin-D-silenced cells (Figure 5F and $5 \mathrm{G})$. Thus, in line with the above pharmacological evidences, the genetic evidences here further confirmed that programmed necrosis pathway mediates GSK1059615-induced cytotoxicity against SCC-9 cells.

\section{GSK1059615 inhibits SCC-9 tumor growth in nude mice, and its activity is compromised with cyclosporin A co-administration}

At last, we studied the anti-tumor activity of GSK1059615 in vivo, via a SCC-9 xenograft nude mice model. As demonstrated, i.p. daily administration of GSK 1059615 at $30 \mathrm{mg} / \mathrm{kg}$ significantly inhibited SCC-9 tumor growth in the nude mice (Figure 6A). Estimated tumor growth, expressed as $\mathrm{mm}^{3}$ per day $[30,31]$, was also dramatically inhibited with GSK1059615 administration (Figure 6B). The weight of GSK1059615-treated tumors was also dramatically lighter than those of vehicle control tumors (Figure 6C). Significantly, as shown in Figure 6A-6C, co-administration of cyclosporin A ( $5 \mathrm{mg} / \mathrm{kg}, i . v$, daily) [32], the cyclophilin-D inhibitor, largely attenuated GSK1059615-induced anti-SCC-9 tumor activity. Thus, mPTP and programmed necrosis pathway may also be required for GSK1059615-induced anti-tumor activity in vivo. Notably, cyclosporin A alone failed to inhibit SCC9 tumor growth in the mice (Figure 6A-6C).Importantly, the mice body weight was not significantly different between the groups (Figure 6D), suggesting that these mice were well-tolerated to the tested regimens. Together, GSK1059615 inhibits SCC-9 tumor growth in nude mice, and its anti-tumor activity in vivo is compromised with co-administration of cyclosporin A.

\section{DISCUSSION}

mTOR lies in the central position in the PI3KAKT-mTOR cascade [5-7]. There are two functionally and structurally distinct mTOR complexes, including the mTOR complex 1 (mTORC1, rapamycin-sensitive) and
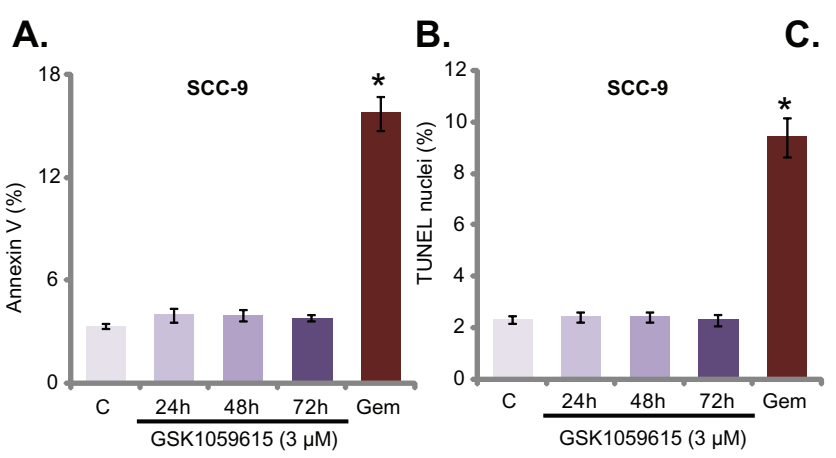

C.

D.
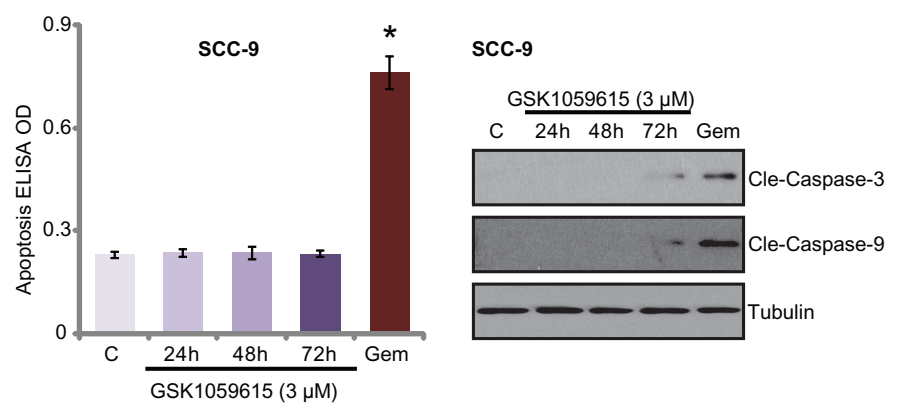

Figure 4: GSK1059615 fails to provoke apoptosis in HNSCC cells. SCC-9 cells were treated with designated GSK1059615 ("GSK") or gemcitabine ("Gem", $500 \mathrm{nM}, 48 \mathrm{~h}$ ), cells were further cultured and cell apoptosis was tested by the listed assays A-C. Expression of caspase proteins was examined by Western blot assay D. For each assay, $n=5$. Experiments in this figure were repeated three times, and similar results were obtained. * $p<0.01$ vs. group " $\mathrm{C}$ ". 
A.

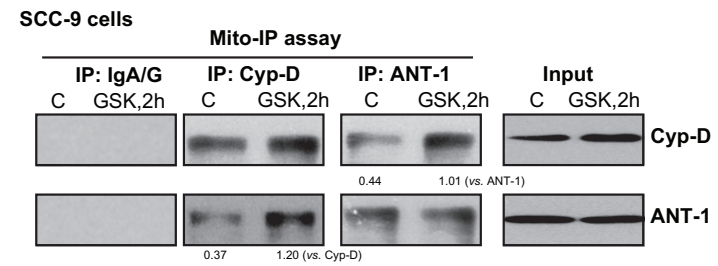

B.

C.

D.
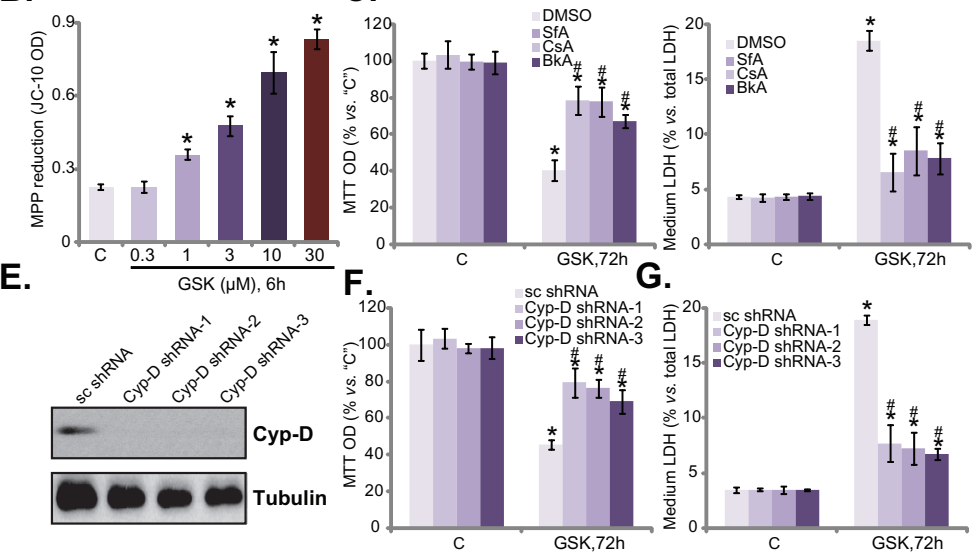

Figure 5: GSK1059615 provokes programmed necrosis in HSNCC cells. SCC-9 cells were treated with GSK1059615 ("GSK", $3 \mu \mathrm{M}$ ) for applied time, mitochondrial lysates were isolated, cyclophilin-D and ANT-1 association was tested by mitochondrial co-immunoprecipitation ("mito-IP") assay (A., data were quantified); Expression of cyclophilin-D ("Cyp-D") and ANT-1 in mitochondrial lysates was also tested as "Input" (A); JC-10 intensity assay was performed to reflect mitochondrial depolarization B. SCC-9 cells, pre-treated for $1 \mathrm{~h}$ with $1.0 \mu \mathrm{M}$ of sanglifehrin A ("SfA"), cyclosporin A ("CsA") or bongkrekic acid ("BkA"), followed by GSK1059615 ("GSK", $3 \mu \mathrm{M}$ ) treatment for 72h, MTT cell viability $\mathbf{C}$. and LDH release D. were tested. SCC-9 cells expressing cyclophilin-D shRNAs ("Cyp-D shRNA1/2/3"), or scramble control shRNA ("sc-shRNA"), were treated with/out GSK1059615 ("GSK", $3 \mu \mathrm{M}, 72 \mathrm{~h}$ ), cyclophilin-D expression E., MTT cell viability F. and LDH release G. were tested. For each assay, $\mathrm{n}=5$. Experiments in this figure were repeated three times, and similar results were obtained. * $p<0.01$ vs. group "C". " $p<0.01$ vs. GSK1059615 only (C and D). " $p<0.01$ vs. "sc-shRNA" (F and G).

A.

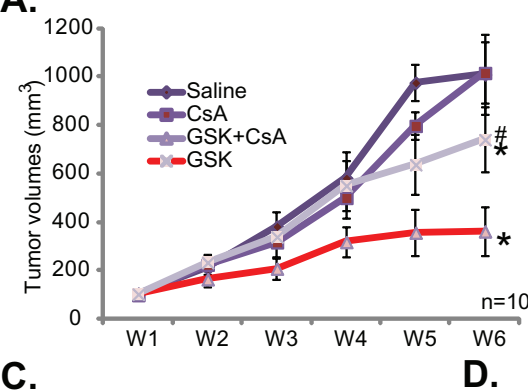

B.
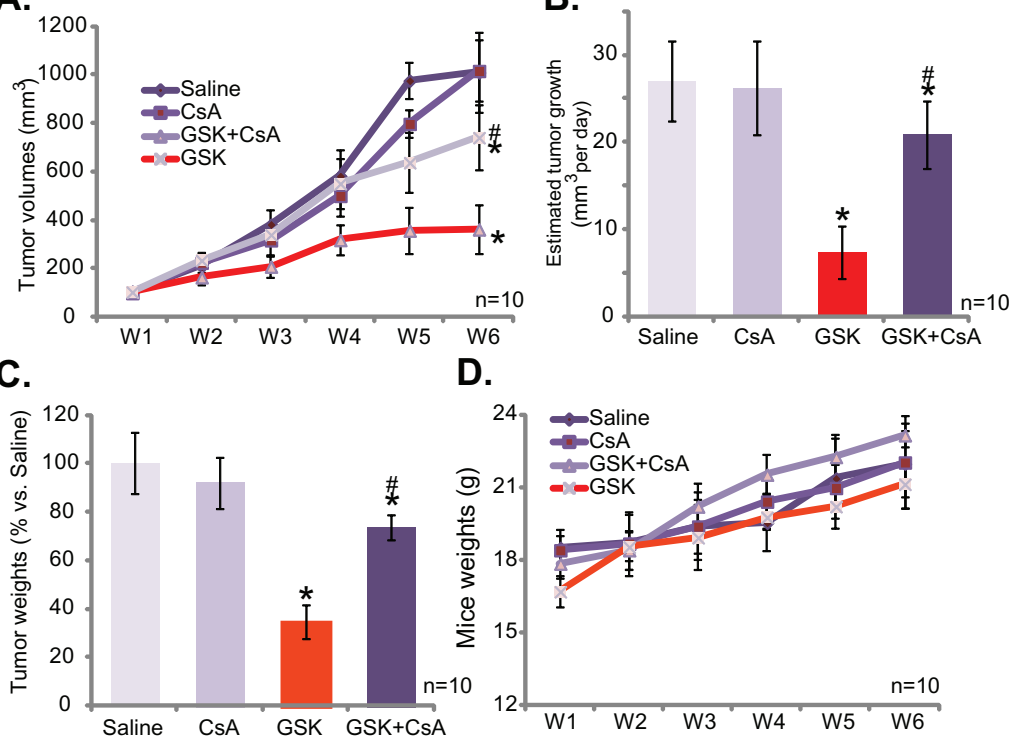

Figure 6: GSK1059615 inhibits SCC-9 tumor growth in nude mice. SCC-9 tumor-bearing nude mice were administrated with GSK1059615 ("GSK", $30 \mathrm{mg} / \mathrm{kg}$, i.p., daily) and/or cyclosporin A ("CsA", $5 \mathrm{mg} / \mathrm{kg}$, i.v., daily), tumor volumes A. and mice body weights D. were recorded every week for a total of 5 weeks; Estimated daily tumor growth was also calculated B.; At the end of experiment, SCC-9 tumors were isolated and weighted $\mathbf{C}$. For each assay, $\mathrm{n}=10$. The data presented were mean $\pm \mathrm{SD}$. ${ }^{*} p<0.01$ vs. group of "Saline". ${ }^{*} p<0.01$ vs. GSK1059615 only. 
mTOR complex 2 (mTORC2, rapamycin-insensitive) [33]. Both mTOR complexes are important for HNSCC cell progression [33]. We found that GSK1059615 not only simantanuously blocked activation of $\mathrm{mTORC} 1$ (indicated by p-S6K1 Thr-389) and mTORC2 (indicated by p-AKT Ser-473), but also inhibited PI3K-AKT activation. This could explain why this compound was more potent in killing HNSCC cells not only than mTOR inhibitors (rapamycin and AZD-2014), but also than the AKT inhibitor (MK-2206 and perifosine). This is also probably why this compound was non-cytotoxic to oral cavity epithelial cells, as these normal cells are with extremely low basal activation of PI3K-AKT-mTOR cascade.

The central function of mitochondrion in regulating intrinsic cell apoptosis has been well-established [34]. Recent studies, interestingly, have proposed the central role of mitochondrion in programming cell necrosis, known as "programmed necrosis" [32, 35-39]. For example, when facing oxidative stresses, p53 will translocate to mitochondrion to trigger $\mathrm{MPTP}$ opening, which leads to necrotic, but not apoptotic, cell death [37]. cyclophilin-D deficiency and mPTP blockers were shown to protect cells and experimental animals from a number of necrotic stimuli, including hypoxia, calcium overload, and oxidative stress [37]. Interestingly, multiple anticancer agents, i.e. cisplatin, doxorubicin, curcumin and icaritin, could also provoke mPTP-dependent programmed necrosis pathway to kill cancer cells [32, 40-42].

In this study, we found that GSK1059615 similarly provoked the programmed necrosis pathway in HNSCC cells, which was evidenced by mitochondria depolarization, ANT-1-cyclophilin-D mitochondrial association and LDH release. Reversely, mPTP blockers or shRNA knockdown cyclophilin-D dramatically alleviated GSK1059615-induced killing of HNSCC cells. In the SCC-9 xenograft tumor nude mice model, coadministration of cyclosporin A significantly attenuated GSK1059615-induced anti-tumor activity. Therefore, targeting PI3K-AKT-mTOR pathway by GSK1059615 possibly provokes programmed necrosis to kill HNSCC cells.

\section{MATERIALS AND METHODS}

\section{Chemicals and reagents}

GSK1059615 was purchased from Adooq Bioscience (Wuxi, China). Gemcitabine and mPTP blockers sanglifehrin A, cyclosporin A and bongkrekic acid as well as wortmannin, LY294002 and perifosine, were provided by Sigma (Shanghai, China). MK-2206, rapamycin and AZD-2014 were purchased from Selleck (Shanghai, China). Antibodies for phospho-PI3K p85 (Tyr458) (\#4228), PI3K p85 (\#4257), p-AKT (Ser 473, \#9271), AKT1 (9272), p-p44/42 MAPK (p-ERK1/2, \#9101), ERK1/2 (\#9102), p-S6K1 (Thr-389, \#9205),
mTOR (\#2983) and p-mTOR (Ser-2448, \#2971) were obtained from Cell Signaling Tech (Shanghai, China). Antibodies for tubulin, adenine nucleotide translocator-1 and cyclophilin-D were obtained from Santa Cruz Biotechnology (Shanghai, China).

\section{Culture of HNSCC cell lines}

SCC-9, SQ20B and A253 cell lines were from Dr. Cui's group [43], and cells were maintained in DMEM medium plus $10 \%$ fetal bovine serum (FBS) [43].

\section{Primary culture human cancer cells and epithelial cells}

Four written-informed consent patients with oral cavity carcinoma (OCC) were enrolled, who received no therapy prior to surgery. OCC tissues and the surrounding normal epithelial tissues were obtained at the time of surgery, and were separated very carefully. As described [43], the tissue specimens were washed and incubated with $0.1 \%$ collagenase I for digestion. Afterwards, cells were filtered via a $70-\mu \mathrm{m}$ nylon cell strainer. Primary cells were cultured in complete DMEM/F12 medium with bFGF and EGF [43]. A total of four lines of primary OCC cells ("OCC1-4") and two lines of oral cavity epithelial cells ("Oepi1-2") were established. Studies requiring human tissues were reviewed and approved by the Institutional Ethics Committee and Internal Review Committee, and were conducted according to Declaration of Helsinki.

\section{MTT assay}

The routine MTT (Sigma) assay was applied to test cell survival with manufactory's recommendation [44].

\section{Clonogenicity assay}

HNSCC cells $\left(1 * 10^{4}\right.$ per $10-\mathrm{cm}$ dish $)$ were trypsinized and suspended in $0.5 \%$ agarose-containing complete medium. Ten days following indicated treatment, the survival colonies were stained and counted manually.

\section{LDH assay}

Cell death was tested via medium release of LDH, using a routine two-step LDH assay kit (Takara, Tokyo, Japan) [45]. LDH in the conditional medium was normalized to the total $\mathrm{LDH}$, reflecting cell necrosis percentage [45].

\section{In vitro proliferation assay}

Cell proliferation BrdU ELISA assay and $\left[\mathrm{H}^{3}\right]$ thymidine incorporation assay were described in detail in other study [18]. The values were always normalized to the untreated control group. 


\section{ELISA assay of cell apoptosis}

The Histone DNA apoptosis ELISA (Roche, Shanghai, China) assay was utilized to quantify cell apoptosis according to the manufacturer's instructions. Detailed protocol can be viewed in other studies [46, 47]. ELISA OD at $450 \mathrm{~nm}$ was utilized to quantify cell apoptosis.

\section{TUNEL assay}

The TUNEL In Situ Cell Death Detection Kit (Roche, Shanghai, China) was utilized to stain nuclei of apoptotic cells. TUNEL ratio of 200 cells per treatment was recorded.

\section{Annexin V FACS assay}

Cells with treatment were incubated immediately with Annexin V-FITC ( $5 \mu \mathrm{g} / \mathrm{mL}$, Invitrogen, Shanghai, China) and the Binding Buffer (Invitrogen), which were subjected to flow cytometry assay of Annexin V.

\section{Detection of mitochondrial depolarization $(\Delta \Psi \mathrm{m})$}

As described previously [29, 35], JC-10 dye assay (Invitrogen) was performed to reflect mitochondrial membrane potential (MMP) reduction. Briefly, following the treatment, cells were stained with JC-10 $(5 \mu \mathrm{g} / \mathrm{mL})$. Cells were then washed, and JC-10 green fluorescence intensity, indicating MMP reduction $(\Delta \Psi \mathrm{m})$, was tested immediately using a fluorescence microplate reader (Titertek Fluoroscan, Germany).

\section{shRNA knockdown of cyclophilin-D}

The three verified lentiviral cyclophilin-D shRNAs (with non-overlapping sequences) and the nonsense scramble shRNA were gifts from Dr. Xu [29]. The lentiviral shRNA was added directly to cells for $12 \mathrm{~h}$. Cells were then subjected to puromycin (5 $\mu \mathrm{g} / \mathrm{mL}$, Sigma) selection for additional 4-5 passages. cyclophilin-D expression in the stable cells was detected by Western blot assay.

\section{Mitochondrial immunoprecipitation (mito-IP) assay}

As described [25], Mitochondria/Cytosol Fractionation Kit (BioVision, Shanghai, China) was utilized to acquire mitochondrial proteins. Six-hundred $\mu \mathrm{g}$ of mitochondrial lysates per treatment were pre-cleared [25]. The lysate supernatant was then rotated overnight with $0.05 \mu \mathrm{g}$ of designated antibody. Next, the protein A/G PLUS-agarose was added to capture the complex.
Pellets were washed, and resuspended in lysis buffer. The immuno-complex was then assayed via Western blot.

\section{Tumor xenograft assay and IHC staining}

Five millions SCC-9 cells per mouse were inoculated s.c. into the female nude mice (5-7 week age, 18-20 grams in weight). Within three weeks, established xenograft tumors were established with volumes around $0.1 \mathrm{~cm}^{3}$. The mice were then randomized into four groups as mentioned in the text ( $\mathrm{n}=10$ per group). Tumor size was measured once every week for a total of 5 weeks, using the modified ellipsoid formula: $(\pi / 6) \times \mathrm{AB}^{2}$, A represents the longest, and $\mathrm{B}$ represents the shortest perpendicular axis of an tumor mass [46]. The animal procedure was approved by the IACUC of authors institutions.

\section{Statistical analysis}

Results were compared by one-way analysis of variance (ANOVA) followed by Turkey's test. Values of $p$ $<0.01$ were considered as statistically significant.

\section{Author contributions}

All authors carried out all the experiments, participated in the design of the project conceived of the study, and participated in its design and coordination and helped to draft the manuscript.

\section{ACKNOWLEDGMENTS}

This work was generously supported by grants from the Wenzhou Science and Technology Bureau (Y20130260). The funders had no role in study design, data collection and analysis, decision to publish, or preparation of the manuscript.

\section{CONFLICTS OF INTEREST}

The authors listed no competing interest.

\section{REFERENCES}

1. Bozec A, Peyrade F, Fischel JL, Milano G. Emerging molecular targeted therapies in the treatment of head and neck cancer. Expert Opin Emerg Drugs. 2009; 14:299-310.

2. Goerner M, Seiwert TY, Sudhoff H. Molecular targeted therapies in head and neck cancer--an update of recent developments. Head Neck Oncol. 2010; 2:8.

3. Sano D, Fooshee DR, Zhao M, Andrews GA, Frederick MJ, Galer C, Milas ZL, Morrow PK, Myers JN. Targeted molecular therapy of head and neck squamous cell carcinoma with the tyrosine kinase inhibitor vandetanib in a mouse model. Head Neck. 2011; 33:349-358. 
4. Peng Y, Zhou Y, Cheng L, Hu D, Zhou X, Wang Z, Xie C, Zhou F. The anti-esophageal cancer cell activity by a novel tyrosine/phosphoinositide kinase inhibitor PP121. Biochem Biophys Res Commun. 2015; 465:137-144.

5. Freudlsperger C, Burnett JR, Friedman JA, Kannabiran VR, Chen Z, Van Waes C. EGFR-PI3K-AKT-mTOR signaling in head and neck squamous cell carcinomas: attractive targets for molecular-oriented therapy. Expert Opin Ther Targets. $2011 ; 15: 63-74$.

6. Molinolo AA, Marsh C, El Dinali M, Gangane N, Jennison K, Hewitt S, Patel V, Seiwert TY, Gutkind JS. mTOR as a molecular target in HPV-associated oral and cervical squamous carcinomas. Clin Cancer Res. 2012; 18:2558-2568.

7. Amornphimoltham P, Patel V, Sodhi A, Nikitakis NG, Sauk JJ, Sausville EA, Molinolo AA, Gutkind JS. Mammalian target of rapamycin, a molecular target in squamous cell carcinomas of the head and neck. Cancer Res. 2005; 65:9953-9961.

8. Carnero A. Novel inhibitors of the PI3K family. Expert Opin Investig Drugs. 2009; 18:1265-1277.

9. Zhang LY, Wu YL, Gao XH, Guo F. Mitochondrial protein cyclophilin-D-mediated programmed necrosis attributes to berberine-induced cytotoxicity in cultured prostate cancer cells. Biochem Biophys Res Commun. 2014; 450:697-703.

10. Qin LS, Jia PF, Zhang ZQ, Zhang SM. ROS-p53cyclophilin-D signaling mediates salinomycin-induced glioma cell necrosis. J Exp Clin Cancer Res. 2015; 34:57.

11. Ouyang L, Shi Z, Zhao S, Wang FT, Zhou TT, Liu B, Bao JK. Programmed cell death pathways in cancer: a review of apoptosis, autophagy and programmed necrosis. Cell Prolif. 2012; 45:487-498.

12. Ju T, Gao D, Fang ZY. Targeting colorectal cancer cells by a novel sphingosine kinase 1 inhibitor PF-543. Biochem Biophys Res Commun. 2016; 470:728-734

13. Guo F, Liu SQ, Gao XH, Zhang LY. AICAR induces AMPK-independent programmed necrosis in prostate cancer cells. Biochem Biophys Res Commun. 2016; 474:277-283.

14. Halestrap AP, McStay GP, Clarke SJ. The permeability transition pore complex: another view. Biochimie. 2002; 84:153-166.

15. Tsujimoto Y, Shimizu S. Role of the mitochondrial membrane permeability transition in cell death. Apoptosis. 2007; 12:835-840.

16. Javadov S, Kuznetsov A. Mitochondrial permeability transition and cell death: the role of cyclophilin d. Front Physiol. 2013; 4:76.

17. Li Y, Cui JT. Inhibition of Bcl-2 potentiates AZD-2014induced anti-head and neck squamous cell carcinoma cell activity. Biochem Biophys Res Commun. 2016; 477:607-13.

18. Zhang YM, Zhang ZQ, Liu YY, Zhou X, Shi XH, Jiang Q, Fan DL, Cao C. Requirement of Galphai1/3-Gab1 signaling complex for keratinocyte growth factor-induced
PI3K-AKT-mTORC1 activation. J Invest Dermatol. 2015; 135:181-191.

19. Hirai H, Sootome H, Nakatsuru Y, Miyama K, Taguchi S, Tsujioka K, Ueno Y, Hatch H, Majumder PK, Pan BS, Kotani H. MK-2206, an allosteric Akt inhibitor, enhances antitumor efficacy by standard chemotherapeutic agents or molecular targeted drugs in vitro and in vivo. Mol Cancer Ther. 2010; 9:1956-1967.

20. Yap TA, Yan L, Patnaik A, Fearen I, Olmos D, Papadopoulos K, Baird RD, Delgado L, Taylor A, Lupinacci L, Riisnaes R, Pope LL, Heaton SP, et al. First-in-man clinical trial of the oral pan-AKT inhibitor MK-2206 in patients with advanced solid tumors. J Clin Oncol. 2011; 29:4688-4695.

21. Van Duyne GD, Standaert RF, Karplus PA, Schreiber SL, Clardy J. Atomic structures of the human immunophilin FKBP-12 complexes with FK506 and rapamycin. J Mol Biol. 1993; 229:105-124.

22. Huo HZ, Zhou ZY, Wang B, Qin J, Liu WY, Gu Y. Dramatic suppression of colorectal cancer cell growth by the dual mTORC1 and mTORC2 inhibitor AZD-2014. Biochem Biophys Res Commun. 2014; 443:406-412.

23. Ying L, Chunxia Y, Wei L. Inhibition of ovarian cancer cell growth by a novel TAK1 inhibitor LYTAK1. Cancer Chemother Pharmacol. 2015; 76:641-650.

24. Kai S, Lu JH, Hui PP, Zhao H. Pre-clinical evaluation of cinobufotalin as a potential anti-lung cancer agent. Biochem Biophys Res Commun. 2014; 452:768-774.

25. Dong YY, Zhuang YH, Cai WJ, Liu Y, Zou WB. The mitochondrion interfering compound NPC-26 exerts potent anti-pancreatic cancer cell activity in vitro and in vivo. Tumour Biol. 2016; 37:15053-15063.

26. Clarke SJ, McStay GP, Halestrap AP. Sanglifehrin A acts as a potent inhibitor of the mitochondrial permeability transition and reperfusion injury of the heart by binding to cyclophilin-D at a different site from cyclosporin A. J Biol Chem. 2002; 277:34793-34799.

27. Basso E, Fante L, Fowlkes J, Petronilli V, Forte MA, Bernardi P. Properties of the permeability transition pore in mitochondria devoid of Cyclophilin D. J Biol Chem. 2005; 280:18558-18561.

28. Tang TS, Slow E, Lupu V, Stavrovskaya IG, Sugimori M, Llinas R, Kristal BS, Hayden MR, Bezprozvanny I. Disturbed $\mathrm{Ca} 2+$ signaling and apoptosis of medium spiny neurons in Huntington's disease. Proc Natl Acad Sci U S A. 2005; 102:2602-2607.

29. Fu M, Shi W, Li Z, Liu H. Activation of mPTP-dependent mitochondrial apoptosis pathway by a novel pan HDAC inhibitor resminostat in hepatocellular carcinoma cells. Biochem Biophys Res Commun. 2016; 477:527-533.

30. Lu PH, Chen MB, Ji C, Li WT, Wei MX, Wu MH. Aqueous Oldenlandia diffusa extracts inhibits colorectal cancer cells via activating AMP-activated protein kinase signalings. Oncotarget. 2016; 7:45889-45900. doi: 10.18632/ oncotarget.9969. 
31. Chen MB, Jiang Q, Liu YY, Zhang Y, He BS, Wei MX, Lu JW, Ji Y, Lu PH. C6 ceramide dramatically increases vincristine sensitivity both in vivo and in vitro, involving AMP-activated protein kinase-p53 signaling. Carcinogenesis. 2015; 36:1061-1070.

32. Zhou C, Chen Z, Lu X, Wu H, Yang Q, Xu D. Icaritin activates JNK-dependent $\mathrm{mPTP}$ necrosis pathway in colorectal cancer cells. Tumour Biol. 2016; 37:3135-44.

33. Laplante M, Sabatini DM. mTOR signaling in growth control and disease. Cell. 2012; 149:274-293.

34. Baines CP. The molecular composition of the mitochondrial permeability transition pore. J Mol Cell Cardiol. 2009; 46:850-857.

35. Zhen YF, Wang GD, Zhu LQ, Tan SP, Zhang FY, Zhou XZ, Wang XD. P53 dependent mitochondrial permeability transition pore opening is required for dexamethasoneinduced death of osteoblasts. J Cell Physiol. 2014; 229:1475-1483.

36. Yu T, Chen C, Sun Y, Sun H, Li TH, Meng J, Shi X. ABT737 sensitizes curcumin-induced anti-melanoma cell activity through facilitating $\mathrm{mPTP}$ death pathway. Biochem Biophys Res Commun. 2015; 464:286-291.

37. Vaseva AV, Marchenko ND, Ji K, Tsirka SE, Holzmann S, Moll UM. p53 opens the mitochondrial permeability transition pore to trigger necrosis. Cell. 2012; 149:1536-1548.

38. Schinzel AC, Takeuchi O, Huang Z, Fisher JK, Zhou Z, Rubens J, Hetz C, Danial NN, Moskowitz MA, Korsmeyer SJ. Cyclophilin D is a component of mitochondrial permeability transition and mediates neuronal cell death after focal cerebral ischemia. Proc Natl Acad Sci U S A. 2005; 102:12005-12010.

39. Qiu Y, Yu T, Wang W, Pan K, Shi D, Sun H. Curcumininduced melanoma cell death is associated with mitochondrial permeability transition pore (mPTP) opening. Biochem Biophys Res Commun. 2014; 448:15-21.
40. Chen B, Xu M, Zhang H, Wang JX, Zheng P, Gong L, Wu GJ, Dai T. Cisplatin-induced non-apoptotic death of pancreatic cancer cells requires mitochondrial cyclophilinD-p53 signaling. Biochem Biophys Res Commun. 2013; 437:526-531.

41. Lu JH, Shi ZF, Xu H. The mitochondrial cyclophilin D/p53 complexation mediates doxorubicin-induced non-apoptotic death of A549 lung cancer cells. Mol Cell Biochem. 2014; 389:17-24.

42. Qiu Y, Yu T, Wang W, Pan K, Shi D, Sun H. Curcumininduced melanoma cell death is associated with mitochondrial permeability transition pore (mPTP) opening. Biochem Biophys Res Commun. 2014; 448:15-21.

43. Li Y, Cui JT. Inhibition of Bcl-2 potentiates AZD-2014induced anti-head and neck squamous cell carcinoma cell activity. Biochem Biophys Res Commun. 2016; 477:607-613.

44. Cao C, Huang X, Han Y, Wan Y, Birnbaumer L, Feng GS, Marshall J, Jiang M, Chu WM. Galpha(i1) and Galpha(i3) are required for epidermal growth factor-mediated activation of the Akt-mTORC1 pathway. Sci Signal. 2009; 2:ra17.

45. Ji F, Mao L, Liu Y, Cao X, Xie Y, Wang S, Fei H. K6PC-5, a novel sphingosine kinase 1 (SphK1) activator, alleviates dexamethasone-induced damages to osteoblasts through activating SphK1-Akt signaling. Biochem Biophys Res Commun. 2015; 458:568-575.

46. Yang YL, Ji C, Bi ZG, Lu CC, Wang R, Gu B, Cheng L. Deguelin induces both apoptosis and autophagy in cultured head and neck squamous cell carcinoma cells. PLoS One. 2013; 8:e54736.

47. Li KR, Zhang ZQ, Yao J, Zhao YX, Duan J, Cao C, Jiang Q. Ginsenoside Rg-1 protects retinal pigment epithelium (RPE) cells from cobalt chloride $(\mathrm{CoCl} 2)$ and hypoxia assaults. PLoS One. 2013; 8:e84171. 